\section{Charge configuration}

Angew. Chem. Int. Ed. http://doi.org/fz9t8r (2012)

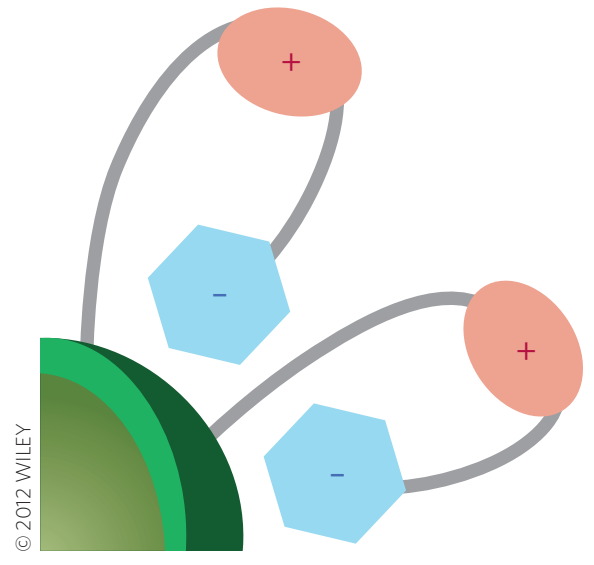

The in vivo behaviour of nanoparticles is known to be dependent on many physical properties including size, shape and charge. Less is understood, however, about the relationship between biological properties and the spatial arrangement of charge on the nanoparticle surface. Now, Han et al. synthesize zwitterionic quantum dots with varying charge distributions and analyse their in vitro and in vivo behaviour. The quantum dots are coated with polymeric ligands containing imidazole units, which bind to the quantum dots in a more chemically stable manner than the commonly used thiol binding group, and either sulphobetaine or carboxybetaine groups. These zwitterionic groups have different binding affinities for the quantum dot surface and hence, result in different charge distributions. Han et al. observe significant nonspecific accumulation of the functionalized quantum dots in the vessels of live mice if positive charges are exposed on the surface but minimal nonspecific accumulation when negative charges are prominent on the surface. This indicates the importance of masking positive charges on the surface of nanomaterials to be used in vivo.

\section{Bulk suppression}

Preprints at http://arxiv.org/abs/1211.6769 and http://arxiv.org/abs/1211.5104 (2012)

The electronic surface states of topological insulators have recently been the subject of intense study by theoreticians and experimentalists alike. A fundamental stumbling block that has so far prevented further inroads being made towards harnessing these surface states for potential applications is the difficulty in distinguishing them from bulk contributions, an irony not lost on many. Zachary Fisk and colleagues present evidence suggesting samarium hexaboride - a so-called Kondo insulator known for decades - is also an ideal topological insulator in which the bulk is completely insulating and the surface highly conducting. Thus, relatively simple transport measurements of the material reveal the properties of the surface conductivity in a manner that has not been possible with other topological insulators such as bismuth selenide. Further work is undoubtedly underway to conclusively prove the origin of these surface states is indeed topological in origin, but what is clear is that there is a new kid on the block for researchers studying topological insulators to focus on.

\section{Hydrogen generation}

Chem. Mater. http://doi.org/j5g (2012)

Dye-sensitized solar cells based on wide-bandgap semiconductor oxides have now been shown to reach solarenergy conversion efficiencies of more

\section{Quantum jumps}

Nano Lett. (In the press); http://doi.org/j5h (2012)

Closely spaced metallic nanoparticles have enabled numerous applications in the fields of plasmonics and metamaterials, including single-molecule sensing, molecular rulers and nanoscale media with tailored optical parameters. Most studies of the plasmonic dimer resonances in such structures have until now been restricted in the classical regime, where the strength of the resonances increases monotonically with decreasing gap between the nanoparticles. Jen Dionne and colleagues have now observed the plasmon resonances of coupled metallic nanoparticles in their full quantum regime for gap sizes reduced to atomic dimensions (subnanometre). Using the electron beam of a scanning transmission electron microscope to manipulate the distance separating 10-nm-diameter spherical nanoparticles and electron energy-loss spectroscopy to observe the dynamic changes of the dimers' resonances, they observed a reduced mode intensity consistent with quantum analyses accounting for the electron tunnelling between the nanoparticles. The full spectral mapping of the dimer-plasmon evolution that the researchers have achieved may open new avenues in the field of quantum nanoplasmonics. than $10 \%$ and are considered to be a promising low-cost alternative to traditional silicon photovoltaic devices. Orthorhombic $\mathrm{Nb}_{2} \mathrm{O}_{5}$ nanocrystalline films functionalized with a RuP compound are now proposed by Thomas Meyer and colleagues as photoanodes in dye-sensitized photoelectrosynthesis cells for hydrogen generation. Compared with anatase $\mathrm{TiO}_{2}$ and with what is generally believed, the conduction band potential for the T-phase orthorhombic $\mathrm{Nb}_{2} \mathrm{O}_{5}$ film is measured to be only slightly higher and is dominated by shallow band-tail trap states. In an operating dye-sensitized photoelectrosynthesis cell, with an ethylenediaminetetraacetic tetra-anion added as a reductive scavenger, hydrogen, quantum yield and photostability measurements show that $\mathrm{Nb}_{2} \mathrm{O}_{5}$ is comparable to $\mathrm{TiO}_{2}$. Overall these results demonstrate that $\mathrm{Nb}_{2} \mathrm{O}_{5}$ is a promising alternative photoanode material for photoelectrochemical applications.

\section{Inclined droplets}

Proc. Natl Acad. Sci. USA http://doi.org/j5f (2013)

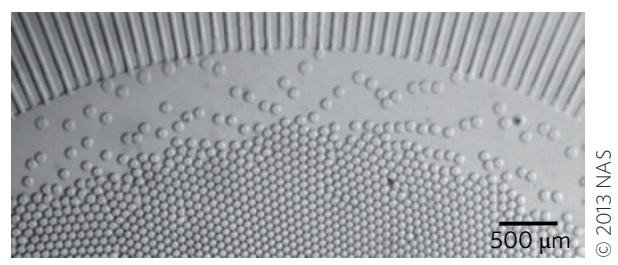

Continuous droplet production is relatively easy: make microchannels with a suitable geometry (such as a T-junction) that allows two fluids (dispersed and carrier) to meet at appropriate flow rates, and hydrodynamic forces will do the rest. However, droplet size and flow rates depend on the viscosities and surface tensions of the two fluids. Now, Rémi Dangla and co-workers report an even simpler approach: inject the dispersed phase through an inlet with a rectangular crosssection into a reservoir with inclined top and bottom walls that is filled with the carrier fluid. Such a gradient in height causes an imbalance of curvature and pressure along the interface between the two fluids, which leads to the formation of a growing 'circular tongue' with a neck of decreasing width in the inlet channel. A droplet forms when the neck ruptures as its width reaches the height of the inlet, and self-propels into the reservoir as a result of the gradient in surface energy imposed by the inclined walls. In this strategy, droplet size depends on the confining geometry only.

Written by Vincent J. Dusastre, Pep Pàmies, Alison Stoddart, Andrea Taroni and Kosmas Tsakmakidis. 